\title{
EULAR definition of arthralgia suspicious for progression to rheumatoid arthritis
}

\author{
Hanna W van Steenbergen, ${ }^{1}$ Daniel Aletaha, ${ }^{2}$ Liesbeth J J Beaart-van de Voorde, ${ }^{1}$ \\ Elisabeth Brouwer, ${ }^{3}$ Catalin Codreanu ${ }_{1}^{4}$ Bernard Combe ${ }_{1}^{5}$ João E Fonseca, ${ }^{6,7}$ \\ Merete L Hetland, ${ }^{8,9}$ Frances Humby, ${ }^{10}$ Tore K Kvien, ${ }^{11}$ Karin Niedermann, ${ }^{12}$ \\ Laura Nuño, ${ }^{13}$ Sue Oliver, ${ }^{14}$ Solbritt Rantapää-Dahlqvist, ${ }^{15}$ Karim Raza, ${ }^{16,17}$ \\ Dirkjan van Schaardenburg, ${ }^{18}$ Georg Schett, ${ }^{19}$ Liesbeth De Smet, ${ }^{1}$ Gabriella Szücs, ${ }^{20}$ \\ Jirí Vencovský, ${ }^{21}$ Piotr Wiland, ${ }^{22}$ Maarten de Wit, ${ }^{23}$ Robert L Landewé, ${ }^{24}$ \\ Annette $\mathrm{H}$ M van der Helm-van Mil ${ }^{1,25}$
}

\section{Handling editor Hans WJ Bijlsma}

- Additional material is published online only. To view please visit the journal online (http://dx.doi.org/10.1136/ annrheumdis-2016-209846)

For numbered affiliations see end of article.

Correspondence to Dr Annette H M van der Helm-van Mil, Department of Rheumatology, Leiden University Medical Center, P.0. Box 9600, Leiden 2300 RC, The Netherlands; AvdHelm@lumc.nl

Received 5 May 2016 Revised 18 August 2016 Accepted 17 September 2016 Published Online First 6 October 2016

\section{CrossMark}

\section{ABSTRACT}

Background During the transition to rheumatoid arthritis (RA) many patients pass through a phase characterised by the presence of symptoms without clinically apparent synovitis. These symptoms are not well-characterised. This taskforce aimed to define the clinical characteristics of patients with arthralgia who are considered at risk for RA by experts based on their clinical experience.

Methods The taskforce consisted of 18 rheumatologists, 1 methodologist, 2 patients, 3 health professionals and 1 research fellow. The process had three phases. In phase I, a list of parameters considered characteristic for clinically suspect arthralgia (CSA) was derived; the most important parameters were selected by a three-phased Delphi approach. In phase II, the experts evaluated 50 existing patients on paper, classified them as CSA/no-CSA and indicated their level of confidence. A provisional set of parameters was derived. This was studied for validation in phase III, where all rheumatologists collected patients with and without CSA from their outpatient clinics.

Results The comprehensive list consisted of 55 parameters, of which 16 were considered most important. A multivariable model based on the data from phase II identified seven relevant parameters: symptom duration $<1$ year, symptoms of metacarpophalangeal (MCP) joints, morning stiffness duration $\geq 60$ min, most severe symptoms in early morning, first-degree relative with RA, difficulty with making a fist and positive squeeze test of MCP joints. In phase III, the combination of these parameters was accurate in identifying patients with arthralgia who were considered at risk of developing RA (area under the receiver operating characteristic curve $0.92,95 \% \mathrm{Cl} 0.87$ to 0.96$)$. Test characteristics for different cut-off points were determined.

Conclusions A set of clinical characteristics for patients with arthralgia who are at risk of progression to RA was established.

\section{INTRODUCTION}

The development of rheumatoid arthritis (RA) is a multistep process. The European League Against Rheumatism (EULAR) study group differentiated the following phases: (1) presence of genetic and environmental risk factors for RA, (2) systemic autoimmunity associated with RA, (3) symptoms without clinical arthritis, (4) unclassified arthritis and finally (5) RA. ${ }^{1}$ The symptomatic phase preceding clinical arthritis is the first opportunity to clinically recognise patients who are at risk for progression to RA. In contrast to the other phases that have been studied extensively, this phase is less well studied. While a few studies reported on symptoms experienced by patients in this phase and on their impact on daily life, ${ }^{2-4}$ clinical characteristics that are specific for this phase have not yet been identified by a consensus-based approach. ${ }^{15} 6$ This situation hampers the conduct of studies and clinical trials in this phase of the disease. It has been shown that early initiation of disease-modifying antirheumatic drug (DMARD) treatment in RA is more effective in modulating the erosive and persisting nature of RA compared with delayed initiation of DMARD treatment. ${ }^{7-9}$ Hence, interventions in the initial clinical phase of the disease, which precedes the onset of clinical arthritis, may be more effective in reducing the risk of disease persistence and the development of damage. ${ }^{10}$ However, studies to address this require the inclusion of homogeneous sets of patients.

Clinical expertise, which includes pattern recognition, guides decisions in daily practice and has also been used as reference for the development of several tools or criteria in the field of rheumatology. ${ }^{11-14}$ Patients with clinically suspect arthralgia (CSA) have articular symptoms without signs of arthritis and are considered to be at increased risk for progression to RA. ${ }^{15}$ Hence, the identification of the presence of CSA is based on clinical expertise. Recent data revealed that patients with CSA constitute only a small percentage of all patients with arthralgia who visit the rheumatology outpatient clinic for the first time $(\sim 7 \%)$, and that a proportion of patients with CSA did indeed progress to RA during follow-up $(\sim 20 \%) .{ }^{16}$ It was also suggested that clinical experience was accurate to distinguish patients with arthralgia at risk of RA from other patients with arthralgia (OR 55). In particular, only a minority of patients who presented 
with arthralgia and subsequently developed RA were not recognised by the rheumatologist. ${ }^{17}$

Although the concept of CSA is appropriate for use in clinical practice, a drawback is its subjectivity, which may result from differences in practice and experience. Therefore, the phenotype of CSA needs to be defined. This taskforce aimed to identify a combination of clinical features that best characterise patients with arthralgia who are at risk of RA according to an expert multidisciplinary group of European rheumatologists, other health professionals and patients. This approach was similar to that which led to the definition of inflammatory back pain, a definition which was subsequently integrated in the Assessment of SpondyloArthritis international Society classification criteria. ${ }^{18} 19$ The taskforce intended to derive a set of clinical parameters to enable the inclusion of homogeneous sets of patients in subsequent studies. It was considered inappropriate to use the phrase 'classification criteria' for the product as, basically, classification concerns testing the presence or absence of a disease. CSA is not in itself a disease, but a combination of symptoms and signs. It was anticipated that clinical characteristics alone are insufficient predictive for RA, that a combination of clinical and other factors (eg, autoantibodies, imaging results) are necessary to identify patients with imminent RA and that the derived clinical definition can later become part of criteria for imminent RA. Thus in sum, the present taskforce aimed to define arthralgia at risk for RA.

\section{METHODS}

\section{Expert committee}

The expert committee comprised 18 rheumatologists, 1 methodologist (RL, who was also one of the rheumatologists), 2 nurse specialists, 1 physiotherapist, 2 patients and 1 research fellow, originating from 15 European countries. The target populations were rheumatologists and health professionals working in secondary care.

\section{Three-phased process}

The process consisted of three phases and two meetings. Expert opinion was the reference. Per phase consensus was obtained before proceeding to the next phase.

\section{Phase I}

Phase I aimed to develop a comprehensive list of clinical parameters (both symptoms at history taking and signs at physical examination) that were considered by the experts to be relevant to distinguish arthralgia that precedes RA from other types of arthralgia. A modified Delphi approach was used. First, all taskforce members were asked to indicate all symptoms and signs that they considered potentially relevant. All parameters mentioned to be relevant by at least two experts or by the patients (based on personal experience) were added to create a comprehensive list. In the next three quantitative rounds, the participants selected the parameters they considered most relevant by weighing. After each round, the list of parameters was modified based on the results; parameters on which consensus was reached (either relevant or irrelevant) were not evaluated in the next round. The group response of the previous round and the modified list were presented to the group before they voted in the next round.

\section{Phase II}

Phase II aimed to develop a provisional set of clinical parameters describing CSA. The experts reviewed clinical data from 50 patients who had previously presented with arthralgia but without clinically detectable arthritis to the rheumatology outpatient clinic of the Leiden University Medical Centre (the Netherlands). Of these, 26 were considered to have CSA by the treating rheumatologist ${ }^{15}$; the prevalence of CSA in this patient set was thus artificial and much higher than in a general rheumatology outpatient clinic. The experts were blinded for grouping by the treating rheumatologists. Clinical data relating to the parameters selected in phase I were presented to the experts as being present or absent in these 'paper patients'. The experts were asked to classify each patient as having CSA or no-CSA and to provide the level of confidence with their classification on a numerical rating scale from 0 (not confident) to 10 (very confident).

Two approaches were used to analyse the data from phase II. First, to gain insight into the degree of equivalence of the expert classifications, the frequencies of the classifications were plotted against the level of confidence of each classification per patient, as described previously. ${ }^{19}$ Individual histograms represented all experts' judgements on individual patient and were evaluated independently by three reviewers (AHMvdH-vM, RLL and HWvS); each reviewer decided whether the experts agreed on the classification as 'CSA', 'no-CSA' or 'unclassifiable'. If all reviewers had the same judgement the patient was categorised accordingly. Otherwise, agreement between the reviewers was reached on how to categorise a patient. The parameters selected in phase I were compared for the patients in the three groups (CSA, no-CSA and unclassifiable). Then, to statistically identify the parameters that best discriminated between CSA and no-CSA, a multilevel model was used with one level being the expert and the other level being the patient; this analysis which was done on 900 judgements about CSA included the data of all 50 patients, each classified by 18 rheumatologists. This mixed effects model with crossed random effects was applied with the weighted CSA classification as outcome and the clinical parameters as independent variables. This model was used to take into account that each expert assessed the same 50 patients. Crossed random effects were included as the symptoms are nested in the combination of expert and patient and thus the residuals of the two levels are still correlated, even after taking the two levels of the analysis into account. ${ }^{20}{ }^{21}$ Clinical parameters with a $\mathrm{p}$ value $\leq 0.05$ in univariable analyses were included in multivariable analysis. The parameters with a positive coefficient in the multivariable analysis were combined to a provisional set of parameters describing CSA. These data were presented at the first meeting.

\section{Phase III}

Phase III aimed to validate the provisional set of parameters in the outpatient clinics of the participating rheumatologists. They were asked to select newly referred patients without a defined time limit of symptoms and without arthritis but with arthralgia who they considered to have an increased risk of RA based on history taking and physical examination (patients with CSA) and patients who had no evident diagnosis or explanation for the arthralgia at first visit but were not considered at risk for RA (no-CSA). Patients who at presentation had evident diagnoses, such as fibromyalgia or osteoarthritis, were not included in the no-CSA group. In addition, the participants were encouraged to base the decision of CSA on the clinical presentation only and not on results of additional investigations. Due to differences in healthcare settings, some rheumatologists had access to the result(s) of laboratory or imaging investigation(s) at first presentation for the majority of their patients. The presence or absence of additional test results at the time of identification of 
CSA or no-CSA was recorded. The provisional set of parameters derived from phase II was tested using multivariable logistic regression analyses in the identified patients with CSA and no-CSA. Thus, again clinical expertise was the reference. The performance of the combination of parameters was assessed using the area under the receiver operating characteristic curve (AUC). Sensitivity and specificity were determined for different cut-off points. The data from this phase were discussed during the second meeting. The final set of parameters was established by voting.

\section{RESULTS}

\section{Phase I: identifying relevant parameters for CSA}

First, all experts identified as many parameters as possible that they considered relevant when evaluating whether patients with arthralgia did or did not have CSA. The total list consisted of 55 parameters (see online supplementary table $\mathrm{S} 1$ ) and included both parameters that were considered to increase and decrease the likelihood of CSA. By selecting and weighing in three rounds, the number of parameters on the list was reduced to 16 (table 1). Consensus was reached to proceed with these 16 parameters to phase II.

\section{Phase II: development of provisional set of parameters describing CSA}

First, in order to get an overview of the data, each of the 50 patients were classified as having CSA, no-CSA or being unclassifiable based on their individual histograms, which represented the classifications of all experts. Seventeen patients were unequivocally classified as no-CSA, 14 as CSA and 19 patients were considered unclassifiable (examples of the histograms are presented in online supplementary figure S1). Table 1 presents the frequencies of the clinical parameters for the groups of patients identified as no-CSA, unclassifiable and CSA.
Then, using data from all 50 patients, a multilevel model with weighted CSA classification as outcome was used to select the parameters that best discriminated between CSA and no-CSA. Results of univariable and multivariable analyses are presented in online supplementary table S2. The following seven variables were presented during the first meeting as a provisional set of parameters describing CSA: joint symptoms of recent onset (duration $<1$ year), symptoms located in metacarpophalangeal (MCP) joints, symmetric symptoms or signs (bilateral in same joint region), duration of morning stiffness $\geq 60 \mathrm{~min}$, most severe symptoms present in the early morning, difficulty with making a fist and positive squeeze test of MCP joints. At the meeting, it was suggested to remove the item symmetry from the multivariable analysis (because of $\mathrm{p}>0.05$ in univariable analysis) and to force metatarsophalangeal (MTP) involvement and a positive family history in the analysis (as these items were judged as very relevant by many experts). The results are presented in online supplementary table S3. Thereafter, consensus was reached on the following seven parameters to characterise arthralgia that is clinically suspect for progression to RA: joint symptoms of recent onset (duration $<1$ year), symptoms located in MCP joints, duration of morning stiffness $\geq 60 \mathrm{~min}$, most severe symptoms present in the early morning, presence of a first-degree relative with RA, difficulty with making a fist and positive squeeze test of MCP joints (box 1).

\section{Phase III: validation}

In total, 322 patients with arthralgia were identified in the different centres (see online supplementary table S4), 142 patients with CSA and 180 patients with arthralgia without CSA. Of them, 78 and 61 respectively were identified based on clinical information only (ie, without data relating to additional investigations); these 139 patients were used in the main analysis. When weighing the parameters based on the B coefficient of the logistic regression analysis after rounding the coefficients to whole points, the combination of seven parameters performed

Table 1 Parameters that were selected in phase I, and frequencies of these parameters in the patients that in phase II were categorised as CSA, no-CSA or were considered unclassifiable

\begin{tabular}{|c|c|c|c|}
\hline & No-CSA $(n=17)(\%)$ & Unclassifiable $(n=19)(\%)$ & $\operatorname{CSA}(n=14)(\%)$ \\
\hline \multicolumn{4}{|l|}{ History taking } \\
\hline Joint symptoms of recent onset (duration $<1$ year) & 41 & 74 & 92 \\
\hline Symptoms in MCP joints & 35 & 63 & 93 \\
\hline Symptoms in MTP joints & 35 & 53 & 57 \\
\hline Symptoms in several small joint regions (MCP, wrists, PIP, MTP joints) & 35 & 68 & 93 \\
\hline Duration of morning stiffness $\geq 60 \mathrm{~min}$ & 6 & 37 & 71 \\
\hline Most severe symptoms in the early morning & 27 & 69 & 90 \\
\hline Improvement of symptoms during the day & 15 & 36 & 90 \\
\hline Increasing number of joints with symptoms over time & 70 & 71 & 90 \\
\hline Patient experience of swelling of small hand joints & 31 & 47 & 77 \\
\hline Positive squeeze test of MCP joints & 14 & 26 & 69 \\
\hline Positive squeeze test of MTP joints & 22 & 21 & 39 \\
\hline
\end{tabular}


Box 1 EULAR defined characteristics describing arthralgia at risk for RA

These parameters are to be used in patients with arthralgia without clinical arthritis and without other diagnosis or other explanation for the arthralgia.

History taking:

- Joint symptoms of recent onset (duration $<1$ year)

- Symptoms located in MCP joints

- Duration of morning stiffness $\geq 60$ min

- Most severe symptoms present in the early morning

- Presence of a first-degree relative with RA

Physical examination:

- Difficulty with making a fist

- Positive squeeze test of MCP joints

EULAR, European League Against Rheumatism; RA, rheumatoid arthritis.

well to explain the clinical expertise (AUC $0.93,95 \%$ CI 0.89 to 0.97$)$. When using all variables unweighted, the combination of seven parameters performed equally well in identifying patients with arthralgia who were considered to be at risk of RA by the experts (AUC 0.92, 95\% CI 0.87 to 0.96) (see online supplementary table S5). The experts agreed that unweighted parameters were more convenient. When analysing all 322 patients, similar AUCs were obtained (see online supplementary table S6).

The sensitivities and specificities belonging to the number of positive parameters are presented in table 2 . A sensitivity $>90 \%$ was obtained in the presence of $\geq 3$ parameters and a specificity $>90 \%$ in the presence of $\geq 4$ parameters. All taskforce members unanimously agreed that arthralgia that is suspected for progression to RA is defined by the seven parameters presented in box 1 and that these parameters are to be used in patients with arthralgia but not with clinical arthritis in whom there is not a better explanation for the arthralgia.

\section{DISCUSSION}

The development of RA is a multistep process. In this project, we defined the combination of symptoms and signs that characterise patients at risk of developing RA. In clinical practice, rheumatologists identify patients with CSA based on their expertise. The presence of CSA may trigger rheumatologists to monitor patients closely and/or to undertake specific laboratory testing or imaging. For daily rheumatological practice, the concept of CSA has been shown to be adequate to differentiate patients with arthralgia, ${ }^{16} 17$

Table 2 Sensitivities and specificities for the presence of arthralgia at risk of RA with the clinical expertise on CSA as reference

\begin{tabular}{lcc}
\hline Number of parameters present & Sensitivity (\%) & Specificity (\%) \\
$\geq 1$ & 100.0 & 14.1 \\
$\geq 2$ & 98.4 & 53.8 \\
$\geq 3$ & 90.2 & 74.4 \\
$\geq 4$ & 70.5 & 93.6 \\
$\geq 5$ & 32.8 & 100.0 \\
$\geq 6$ & 16.4 & 100.0 \\
$\geq 7$ & 1.6 & 100.0 \\
\hline
\end{tabular}

CSA, clinically suspect arthralgia; RA, rheumatoid arthritis. but it is subjective and this results in heterogeneity. For scientific studies, homogeneous sets of patients are required. Therefore, we aimed to capture clinical expertise and represent it in a set of defined clinical parameters. The process incorporated three phases and two meetings, and the product was obtained by a data-driven and consensus-driven approach. Unanimous agreement was obtained on seven parameters reflecting the aggregated expertise of rheumatologists, healthcare professionals and patients from 15 European countries.

This taskforce was able to successfully identify and collate a homogenous and measurable set of clinical parameters of CSA based on clinical expertise of rheumatology experts for use in future studies. Further longitudinal studies are required to assess if this definition reduces the number of patients with arthralgia that need additional testing, and to determine the predictive accuracy of these clinical parameters for the development of RA, both when used alone and in combination with the results of additional investigations. Thus, the result of this taskforce should serve as the basis for the next step, which is the initiation of longitudinal data-driven projects, which ultimately results in the development of criteria for imminent RA. Most likely, such criteria will include both clinical-based and investigation-based parameters (such as laboratory and imaging results).

Because a clinical definition alone is unlikely to be sufficiently accurate to identify patients with RA in a symptomatic prearthritis phase, and because CSA is not a disease but the description of a phenotype, it was decided that the product of this taskforce should not be referred to as 'classification criteria' but as a 'definition'. Furthermore, while the physicians in the taskforce argued that the word 'patient' may have an unwarranted connotation, the patient representatives in the task force justified the use of the term 'patient' by pointing to the fact that these individuals had presented with pain and other symptoms and had been referred to secondary care.

The parameters characterising arthralgia at risk of RA may serve as the basis for observational studies and intervention trials performed in the symptomatic pre-arthritis phase. Depending on the study, a more sensitive or more specific definition may be preferred. A high sensitivity may be preferred if the clinical criteria are used as first inclusion criterion, as in this situation the number of patients with CSA that are missed by the criteria should be low. Subsequently, additional tests can be applied to ensure sufficient specificity. If in contrast, patients are mainly selected based on clinical characteristics, a higher specificity may be preferred to prevent false-positives. Given this, the taskforce deliberately avoided a single cut-off point to define arthralgia at risk of RA, but provided the test characteristics of a spectrum of cut-off points. A high sensitivity $(>90 \%)$ is obtained if $\geq 3$ out of the 7 parameters are present; a high specificity (>90\%) requires the presence of $\geq 4$ of the 7 parameters.

The clinical variables were considered to distinguish patients with arthralgia who are at risk of RA from patients with other types of (not specified) arthralgia. Patients who at first presentation clearly had other diagnoses, such as fibromyalgia or osteoarthritis, were not included in the control groups of phases II and III. This is in line with clinical practice, as there is no diagnostic dilemma in the patients with evident diagnoses. Similar to the 2010 American College of Rheumatology/EULAR classification criteria for RA that should not be applied to patients with arthritis with diagnoses other than RA, ${ }^{14}$ the present set of parameters is reserved for patients with arthralgia with no definitive diagnosis but a clinical suspicion of RA.

The definition was derived for use in secondary care. Because of this target population, the taskforce was composed largely of 
rheumatologists and their expertise was used as a reference. General practitioners were not involved. The taskforce discussed whether our present product may be useful as a referral tool for general practitioners, as has been done by others. ${ }^{22}$ While the taskforce was of the opinion that the present set of parameters might also be valuable to identify patients with arthralgia at risk of RA in primary care, it was agreed that the applicability of the present definition in the primary care setting would need to be assessed through future research in primary care.

It was acknowledged that there may be some redundancy in the seven parameters expressing risk for RA. Further prospective studies will be required to elucidate if one of the parameters can be omitted without losing discriminative ability.

A limitation of our approach is that the experts who developed the list of relevant parameters in phase I and scored the patients in phase II also identified patients for the validation phase. It is possible that the discussions that were held and the data from the first two phases influenced their clinical expertise while selecting patients with CSA and patients with arthralgia without CSA. However, many experts also involved other colleagues to select patients with CSA from their clinics and these colleagues were not involved in the first two phases of the project.

Differences in healthcare settings affect the ability to identify patients in a symptomatic phase prior to presenting with clinically apparent arthritis. For example, between centres and countries there are differences in the possibilities for early access. Some of the differences between healthcare settings were incorporated by inviting experts from different centres and different countries and by using a consensus-based approach. There were also differences in the extent to which additional investigations were performed prior to the first clinical evaluation in specialty care. As the aim of the taskforce was to provide a clinical definition, and as knowledge of the results of additional investigations may influence the selection of patients in phase III, patients in whom knowledge of additional investigations were known at first presentation were initially excluded from analyses. This ensured that patients were exclusively identified on the clinical presentation. However, a subanalysis including also the other patients did not give different results, revealing robustness of the data.

The taskforce had discussed if the individual parameters needed to be defined. Consensus was derived that this project was not aiming at what definition of a specific domain was best, but rather what domains contribute most to the 'phenotype' of CSA, given all the restrictions.

In conclusion, a set of clinical characteristics describing arthralgia at risk of RA was established. The combination of these parameters accurately reflected expert opinion about CSA. Test characteristics were determined for different cut-off points. For a sensitive definition, arthralgia at risk of RA can be defined by the presence of $\geq 3$ parameters and the presence of $\geq 4$ parameters yielded a high specificity. Longitudinal studies are required to determine the predictive accuracy of these clinical parameters alone and when combined with the results of additional investigations, such as laboratory testing or imaging.

\footnotetext{
Author affiliations

${ }^{1}$ Department of Rheumatology, Leiden University Medical Center, Leiden, The Netherlands

${ }^{2}$ Department of Internal Medicine 3, Division of Rheumatology, Medical University Vienna, Vienna, Austria

${ }^{3}$ Department of Rheumatology and Clinical Immunology, University of Groningen, University Medical Center, Groningen, The Netherlands

${ }^{4}$ Rheumatology Department, Center of Rheumatic Diseases, Bucharest, Romania

${ }^{5}$ Department of Rheumatology, Montpellier University Hospital, Montpellier, France
}

${ }^{6}$ Rheumatology Research Unit, Instituto de Medicina Molecular, Faculdade de Medicina da Universidade de Lisboa, Lisbon, Portugal

${ }^{7}$ Rheumatology Department, Centro Hospitalar de Lisboa Norte, EPE, Hospital de Santa Maria, Lisbon Academic Medical Centre, Lisbon, Portugal

${ }^{8}$ DANBIO registry and Center for Rheumatology and Spine Diseases, Centre of Head and Orthopaedics, Rigshospitalet, Copenhagen, Denmark

${ }^{9}$ Department of Clinical Medicine, Faculty of Health and Medical Sciences, University of Copenhagen, Copenhagen, Denmark

${ }^{10}$ Queen Mary University of London, Centre for Experimental Medicine and

Rheumatology, William Harvey Research Institute, London, UK

${ }^{11}$ Department of Rheumatology, Diakonhjemmet Hospital, Oslo, Norway

${ }^{12}$ Institute of Physiotherapy, School of Health Professions, Zurich University of

Applied Sciences, Winterthur, Switzerland

${ }^{13}$ Department of Rheumatology, Hospital Universitario La Paz-IdiPaz, Paseo de La Castellana, Madrid, Spain

${ }^{14}$ Independent Nurse Consultant, North Devon, UK

${ }^{15}$ Department of Public Health and Clinical Medicine/Rheumatology, University

Hospital, Umeå, Sweden

${ }^{16}$ Rheumatology Research Group, Institute of Inflammation and Ageing, College of

Medical and Dental Sciences, University of Birmingham, Birmingham, UK

${ }^{17}$ Sandwell and West Birmingham Hospitals NHS Trust, Birmingham, UK

${ }^{18}$ Amsterdam Rheumatology and immunology Center, locations Reade and Academic Medical Center, Amsterdam, The Netherlands

${ }^{19}$ Department of Internal Medicine 3, University of Erlangen-Nuremberg, Erlangen, Germany

${ }^{20}$ Department of Rheumatology, University of Debrecen Medical Center, Debrecen, Hungary

${ }^{21}$ Department of Rheumatology, First Faculty of Medicine, Institute of Rheumatology, Charles University, Prague, Czech Republic

${ }^{22}$ Department of Rheumatology and Internal Medicine, Wroclaw Medical University, Wroclaw, Poland

${ }^{23}$ EULAR Standing Committee of People with Arthritis/Rheumatism in Europe (PARE), Zurich, Switzerland

${ }^{24}$ Amsterdam Rheumatology and Immunology Center, Amsterdam, the Netherlands and Atrium Medical Center, Heerlen, The Netherlands

${ }^{25}$ Department of Rheumatology, Erasmus University Medical Center, Rotterdam, The Netherlands

Acknowledgements The authors acknowledge Sofia Ramiro for her help with the statistical analysis.

Contributors All authors contributed substantially to the conception and design of the work and acquisition and interpretation of the data. HWvS, AHMvdH-vM and

RLL additionally analysed the data and drafted the initial version of the manuscript All other authors revised the manuscript critically for important intellectual content and all authors gave approval of the final version to be published.

Funding This taskforce developing the presented definition was financially supported by EULAR Standing Committee on Clinical Affairs (ESCCA).

Competing interests None declared.

Patient consent Obtained.

Ethics approval The medical ethics committee of the Leiden University Medical Centre obtained approval for the patient information used in phase I and phase II. In phase III, approval was obtained, if necessary, according to the local guidelines of the participating centres.

Provenance and peer review Not commissioned; externally peer reviewed

\section{REFERENCES}

1 Gerlag DM, Raza K, van Baarsen LG, et al. EULAR recommendations for terminology and research in individuals at risk of rheumatoid arthritis: report from the Study Group for Risk Factors for Rheumatoid Arthritis. Ann Rheum Dis 2012;71:638-41.

2 Stack RJ, van Tuyl LH, Sloots M, et al. Symptom complexes in patients with seropositive arthralgia and in patients newly diagnosed with rheumatoid arthritis: a qualitative exploration of symptom development. Rheumatol Oxf Engl 2014;53:1646-53.

3 van Tuyl LH, Stack RJ, Sloots M, et al. Impact of symptoms on daily life in people at risk of rheumatoid arthritis. Musculoskeletal Care 2016;14:169-73.

4 Newsum EC, van der Helm-van Mil AH, Kaptein AA. Views on clinically suspect arthralgia: a focus group study. Clin Rheumatol 2016:35:1347-52.

5 van Steenbergen HW, Huizinga TW, van der Helm-van Mil AH. Review: the preclinical phase of rheumatoid arthritis: what is acknowledged and what needs to be assessed? Arthritis Rheum 2013;65:2219-32.

6 Raza K, Gerlag DM. Preclinical inflammatory rheumatic diseases: an overview and relevant nomenclature. Rheum Dis Clin N Am 2014;40:569-80.

7 Finckh $\mathrm{A}$, Liang $\mathrm{MH}$, van Herckenrode $\mathrm{CM}$, et al. Long-term impact of early treatment on radiographic progression in rheumatoid arthritis: a meta-analysis. Arthritis Care Res 2006;55:864-72. 
8 van der Linden MP, le Cessie S, Raza K, et al. Long-term impact of delay in assessment of patients with early arthritis. Arthritis Rheum 2010;62:3537-46.

9 van Nies JA, Krabben A, Schoones JW, et al. What is the evidence for the presence of a therapeutic window of opportunity in rheumatoid arthritis? A systematic literature review. Ann Rheum Dis 2014;73:861-70.

10 Mankia K, Emey P. Preclinical rheumatoid arthritis. Progress toward prevention. Arthritis Rheumatol 2016;68:779-88.

11 van der Heijde DM, van 't Hof MA, van Riel PL, et al. Judging disease activity in clinical practice in rheumatoid arthritis: first step in the development of a disease activity score. Ann Rheum Dis 1990;49:916-20.

12 Fautrel B, Combe B, Rincheval N, et al. Level of agreement of the 1987 ACR and 2010 ACR/EULAR rheumatoid arthritis classification criteria: an analysis based on ESPOIR cohort data. Ann Rheum Dis 2012;71:386-9.

13 Gärtner M, Fabrizii JP, Koban $\mathrm{E}$, et al. Immediate access rheumatology clinic: efficiency and outcomes. Ann Rheum Dis 2012;71:363-8.

14 Aletaha D, Neogi T, Silman AJ, et al. 2010 Rheumatoid arthritis classification criteria: an American College of Rheumatology/European League Against Rheumatism collaborative initiative. Ann Rheum Dis 2010;69:1580-8.

15 van Steenbergen HW, van Nies JA, Huizinga TW, et al. Characterising arthralgia in the preclinical phase of rheumatoid arthritis using MRI. Ann Rheum Dis 2015;74:1225-32.
16 van Steenbergen HW, Mangnus L, Reijnierse M, et al. Clinical factors, anticitrullinated peptide antibodies and MRI-detected subclinical inflammation in relation to progression from clinically suspect arthralgia to arthritis. Ann Rheum Dis 2016;75:1824-30.

17 van Steenbergen HW, van der Helm-van Mil AH. Clinical expertise and its accuracy in differentiating arthralgia patients at risk for rheumatoid arthritis from other patients presenting with joint symptoms. Rheumatol Oxf Engl 2016;55:1140-1.

18 Sieper J, van der Heijde D, Landewé R, et al. New criteria for inflammatory back pain in patients with chronic back pain: a real patient exercise by experts from the Assessment of SpondyloArthritis international Society (ASAS). Ann Rheum Dis 2009;68:784-8.

19 Rudwaleit M, Landewé R, van der Heijde D, et al. The development of Assessment of SpondyloArthritis international Society classification criteria for axial spondyloarthritis (part I): classification of paper patients by expert opinion including uncertainty appraisal. Ann Rheum Dis 2009;68:770-6.

20 Rabe-Hesketh S, Skrondal A. Multilevel and longitudinal modeling using Stata. 2nd edn. Stata Press, 2008. ISBN-13 978-1-59718-040-5

21 Quené $H$, van den Bergh $H$. Examples of mixed-effects modeling with crossed random effects and with binomial data. J Mem Lang 2008;59:413-25.

22 Bell MJ, Tavares R, Guillemin F, et al. Development of a self-administered early inflammatory arthritis detection tool. BMC Muscoloskelet Disord 2010;11:50. 